\title{
LA ENFERMEDAD DE TODOS EN EL CUERPO PROPIO: BRUJERÍA Y PERFORMATIVIDAD DEL TRIBUNAL DE LA RAZA INDÍGENA EN CHILOE ${ }^{1}$
}

\section{The embodiment of reciprocity: Sourcery and performativity in the Tribunal de la Raza Indigena in Chiloé}

Fernando A. Valenzuela*

\section{RESUMEN}

Discursos dieron forma e hicieron real la asociación de brujos Tribunal de la Raza Indígena, también llamada Recta Provincia, en Chiloé a fines del siglo diecinueve. Tales discursos, recogidos en las actas del proceso judicial realizado contra individuos que fueron seńalados como brujos en Chiloé en 1880, presuponen la distinción entre una dimensión inmanente y una dimensión transcendente del mundo, y una comprensión del cosmos como estando gobernado por la forma de la reciprocidad. La desilusión de las expectativas de reciprocidad en las cuales se sostenía el sistema social se podía manifestar como enfermedad en los cuerpos de los individuos. Esto daba lugar a la utilización de técnicas mágicas y de adivinación para compensar, por medio de la venganza, el quiebre de expectativas normativas de comportamiento.

Palabras clave: Magia, brujería, pensamiento tradicional, cosmovisión, performatividad, Teoría de sistemas, Chiloé.

\footnotetext{
${ }^{1}$ Artículo realizado en el marco del Proyecto $\mathrm{N}^{\circ} 1121124$ del Fondo Nacional de Desarrollo Científico y Tecnológico (FONDECYT), titulado: "Datos y relatos científico sociales que dan forma a la realidad social de Chile: Estudio de los entrelazamientos constructivos y performativos de la ciencia social”.

Agradezco las observaciones que Claudio Ramos, Cecilia Bralic, Darío Rodríguez e Ignacio Farías, hicieran a versiones previas de este texto.

*Escuela de Sociología, Universidad Andres Bello. Viña del Mar, Chile. Correo electrónico: fernando.valenzuela@unab.cl Artículo recibido el 7 de enero de 2013. Aceptado el 26 de agosto de 2013.
} 


\begin{abstract}
The records of the legal process undertaken against individuals that were signaled as practitioners of witchcraft in Chiloé at the end of the nineteenth century give account of discourses that, by sustaining a world in which its existence is plausible, created and realized an association of witchcraft, called Tribunal de la Raza Indígena, also known as the Recta Provincia. These discourses presuppose a distinction between an immanent and a transcendental dimension of the world, and a cosmos governed by laws of reciprocity. The Tribunal de la Raza Indígena, as it appears in these records, may be understood as an association of witches that used magical and divinatory techniques to compensate, through vengeance, episodes of violation of normative behavioral expectations that characterize reciprocal social relations. Such episodes were manifested in the form of sickness in the bodies of individuals. This gave way to the use of magical and divinatory techniques to compensate, through vengeance, the rupture of normative expectations of behavior.
\end{abstract}

Keywords: Magic, witchcraft, traditional thought, world view, performativity, systems theory, Chiloé.

\title{
INTRODUCCIÓN
}

Es conocido el episodio legal ocurrido en 1880 en la provincia de Chiloé, que fuera comunicado por Ramón Espech a Benjamín Vicuña Mackenna:

"Desde tiempos inmemoriales los brujos de Chiloé y su Cueva de Quicaví gozaron en todo el país de renombre no exento de temores y cuidados, pero en el último cuarto del siglo XIX sus actividades no se limitaron a simples actos de hechicería sino que decididamente se dedicaron a asesinar por cuenta propia y ajena, extendiéndose el terror en todas las islas y comarcas del Archipiélago de Chiloé. Semejante situación movió en 1880 al Intendente de la Provincia don Luis Martianino Rodríguez, funcionario relevante por su inteligencia, capacidad y energía, a tomar las medidas necesarias para ponerle término. Ordenó una redada de todos los individuos señalados como brujos; concentrólos en Ancud y aquellos que parecieron culpables de delitos comunes fueron entregados a la acción de la justicia" (Espech, 1960: 124).

$\mathrm{Al}$ menos desde esta comunicación de Ramón Espech, varios autores se han ocupado de organizar comunicaciones sobre los brujos de Chiloé. Como resultado, contamos con detallados diccionarios de roles, procedimientos, instrumentos y síntomas de la brujería chilota, redactados la mayoría de las veces con una mirada de soslayo que no sabe si aceptar como verdadero este conocimiento sobre cadenas 
causales que se internan en un espacio inaccesible para los no iniciados (Álvarez Sotomayor, 1954; Balbontín Moreno, 1965; Cavada, 1914; Contreras, 1966; León, 2007; Romo Sánchez, 1987, 1989, 1997). Con razón Francisco Cavada incluye antídotos y precauciones: "El estornudar un visitante al echarse un puñado de afrecho al fuego, es indicio de que es brujo" (Cavada, 1914: 111); "El Brujo es invulnerable contra cualquier arma de fuego, excepto contra la escopeta cargada con sal bendita" (Cavada, 1914: 109).

Las actas de los procesos legales realizados a quienes fueron señalados como brujos a finales del siglo XIX, publicadas en los Anales Chilenos de Historia de la Medicina (Espech, 1960), enriquecieron significativamente esta documentación, permitiendo construir narraciones históricas sobre la trayectoria y los procedimientos del que se llamara -entre otros nombres- "Tribunal de la Raza” (Anónimo, 1908; Barrientos, 1988; Cárdenas Saldivia, 1989; Espech, 1960; Mancilla Pérez, 1994a, 1994b; Marino y Osorio, 1987; Rojas F., 2002).

Gonzalo Rojas F. (2002: 60-66) ofrece una síntesis de este corpus bibliográfico junto con interesantes hipótesis sobre los orígenes y la evolución de esta asociación de brujos. Como contexto argumental, distingue dos principales clases de hipótesis acerca del origen colonial de la asociación de brujos en Chiloé. De un lado, argumentos funcionalistas han intentado dar cuenta de la asociación de brujos de Chiloé en términos de un mecanismo poco diferenciado que contrapesara la debilidad de instituciones especializadas en la aplicación de la ley y en la salud (Flores Ábalos 1994; citado por Rojas F., 2002). Desde una tradición de teoría sociológica funcionalista centrada en los problemas de integración social, las asociaciones de brujos corresponderían a mecanismos propios de sociedades "simples" - es decir, sociedades con bajos niveles de diferenciación interna o de especialización funcional de sus instituciones (Parsons, 1971, 1974)- que sirven de equivalentes funcionales (Merton, 1996) de instituciones "modernas" especializadas en los ámbitos de la normatividad y de la salud. Desde el punto de vista de las teorías sociológicas del conflicto, por su parte, esta asociación de brujos ha sido comprendida también como una forma de resistencia indígena a la dominación española en distintos flancos: político-legal, religioso y lingüístico, permitiendo la supervivencia de tradiciones autóctonas (Cárdenas, Montiel y Hall, 1991: 26).

Frente a ambas alternativas, y asumiendo una perspectiva de procesos históricos, Gonzalo Rojas F. ha argumentado que "La Mayoría" -otro de los 
nombres con que los documentos se refieren a la asociación de brujos de Chiloéemergió en el siglo dieciocho como consecuencia del proceso de evangelización que destruyó el sistema chamánico:

"Con la persecución de las prácticas y ritos mágicos, la desaparición de las ceremonias religiosas, el desplazamiento de los machis y, en términos generales, con la destrucción del sistema chamánico, el cristianismo potenció indirectamente a los hechiceros. Estos no sólo aumentaron su poder e influencia sobre las comunidades indígenas, sino que asumieron además nuevas funciones, tanto en el plano de los ritos mágico-curativos, de fertilización y de adivinación como en el de la autorregulación de la brujería y la administración de la justicia” (Rojas F., 2002: 59).

Según esta línea de argumentación desarrollada por Gonzalo Rojas F., los hechiceros (o brujos) fueron los herederos de los kalku de la tradición mapuchehuilliche. Al contrario que los machi, los kalku manipulaban los espíritus relacionados con el principio del mal (wekufü), siéndoles atribuida la autoría de los males que los machi podían luego curar. Durante el período colonial el principio del mal o wekufü habría sido identificado con el demonio, y con ello los kalku habrían sido identificados con los brujos o hechiceros de la tradición occidental. De tal manera, el "Tribunal de la Raza," "La Recta Provincia" o "La Mayoría” es descrita en los documentos de la época y en la bibliografía especializada como una asociación de tales brujos. Siguiendo al mismo autor, no habría sido sino hasta el siglo dieciocho, con la debilitación de la posición de los machi, que tal asociación habría logrado centralidad.

En la distinción machi/kalku se anuncia ya un rasgo fundamental de la imagen de mundo que reproducen los documentos sobre la asociación de brujos: el que “...en los génesis de toda enfermedad siempre estaba la voluntad de algún hombre o espíritu que quería hacer daño" (Rojas F., 2002: 22). La enfermedad se padece como el fin buscado por la acción de alguien, de modo que las miradas se dirigen hacia el sujeto que lleva a cabo la acción, sus fines y sus medios. Ello implica que la correcta identificación de quiénes eran kalku habría sido una preocupación central en el proceso de sanación, pues habría permitido esclarecer la causa del mal. Tal investigación se realizaba por medio de prácticas de adivinación, las cuales componen la mayor parte de las actas correspondientes al proceso legal que se llevó a cabo contra quienes fueron señalados como brujos en Chiloé en 1880.

La transcripción de tal proceso fue publicada por Ramón Espech en los Anales de Historia de la Medicina en 1960. Una revisión de este material nos 
permite observar esta asociación, y la observación que de ella hacían las autoridades republicanas, desde un punto de vista complementario al de Rojas. En este artículo me interesa analizar este proceso en términos de un modo particular de enfrentar normativamente el problema de la contingencia e inestabilidad de las estructuras sociales entendidas como expectativas de comportamiento (Luhmann, 1995:315), que se contrapone al modo que es característico de la institución que la observa y toma nota de sus procesos en términos de la diferencia entre legalidad e ilegalidad, pudiendo ser entendida como un sistema funcional del derecho (Luhmann, 2002). Los procesos propios de la asociación de brujos, según son descritos por las actas judiciales, son vistos aquí una vez más como "mecanismos” y, en cuanto tales, como procesos que llevan a cabo una "función" social: la institución y sus procesos son analizados en calidad de "equivalentes funcionales" a la luz de problemas del sistema social en el cual se reproduce. Es éste, por lo tanto, un análisis cercano a la hipótesis funcionalista reconocida por Rojas, pero que puede integrar la hipótesis de Rojas en términos de "avances preadaptativos" que, frente a cambios en el entorno, se han convertido en "avances selectivos" (Luhmann, 1997: 512). Es decir, la distinción machi/kalku puede ser vista como una condición que ha sido aprovechada en un proceso evolutivo recursivo para enfrentar nuevos problemas societales; como un parásito que ha logrado centralidad en una nueva situación social gobernando nuevas posibilidades de cambio (Serres, 2007).

La hipótesis que desarrollaré a lo largo de este artículo se concentra del lado del Tribunal de la Raza Indígena. Aquí nos encontramos con la descripción de una asociación que utilizaba técnicas mágicas y de adivinación para compensar, por medio de la venganza, episodios de desilusión de las expectativas de comportamiento en las que descansan las relaciones de reciprocidad.

El modo de operar de esta institución, tal como es descrita en los documentos disponibles, asume una representación del mundo que es concordante con una sociedad segmentada que no ha adoptado la escritura como medio de comunicación (Cevolini, 2011; Luhmann, 1997): un mundo estructurado en base a la distinción entre un ámbito asequible (familiar y conocido) y otro inasequible (no familiar y desconocido). Las técnicas de adivinación se basan en la comprensión del mundo inmediato, familiar, como una red de signos cuyo sentido proviene del dominio desconocido e inasequible del mundo: superficies cuyos movimientos reflejan los movimientos que ocurren en el fondo. Aquel orden del cosmos, ese fondo cuyos movimientos son reflejados por los eventos de la cotidianidad, es 
fundamentalmente el orden de la reciprocidad, como fuera conceptualizado por Marcel Mauss (2007). Este es un orden que, como observara Niklas Luhmann, asegura en el nivel de las estructuras sociales la equivalencia entre los sistemas parciales de las sociedades diferenciadas según un criterio de segmentación, al desplazar temporalmente las diferencias entre ellos por medio de una promesa de devolución (Luhmann, 1997). De tal modo, se trata de una sociedad que observa sus propias estructuras por medio de la atribución de éstas a un orden externo.

La magia, por su parte, “...ofrece la posibilidad de paralelizar en lo desconocido las causalidades conocidas mediante prácticas que, a su vez, están disponibles como conocidas" (Luhmann y De Giorgi, 1998: 299). Por medio de técnicas mágicas no sólo se busca establecer el sentido profundo de los eventos superficiales, como en la adivinación, sino que se busca introducir diferencias en la superficie a través de la manipulación de las cadenas causales que la organizan del lado desconocido del mundo. En el sentido de Marcel Mauss (2001), se entiende aquí la magia como una institución netamente performativa o realizativa (Austin, 2008; Callon, 2007; Ramos Zincke, 2012), que "hace cosas" o tiene efectos en la realidad, usualmente por medio de la intervención de elementos de un orden de realidad distinto a la conducta ritual misma, y que no forma parte de cultos organizados, siendo relegada a un ámbito privado y secreto.

Las actas describen una colectividad de brujos que develan y manipulan tales cadenas causales en el dominio de lo inasequible, y con ello refuerzan expectativas de reciprocidad al interior de la comunidad. Esto implica que los efectos de la magia, si bien se verifican concretamente en el cuerpo de las personas, se ramifican en el "cuerpo social" en la forma de expectativas normativas de comportamiento, entendiendo éstas como aquellas expectativas que no son corregidas en caso de desilusión, pues se las tiene por justificadas (Luhmann, 1996, 2007).

Si eso es lo que se observa cuando se pone la mirada en el referente objetivo de los documentos sobre la asociación de brujos -en la asociación misma como institución histórica-, las operaciones sociales que sostienen este mundo son más claras cuando, en lugar de mirar a través de los documentos, cual ventana hacia la realidad histórica, permanecemos en el nivel del discurso. Aquí se tiene que todos los individuos interrogados a lo largo del proceso judicial informado por Ramón Espech dan cuenta de su participación en la institución de la brujería al afirmar y dar sostén, en sus discursos y prácticas, a un mundo en el cual esta institución puede existir. Y en este sentido sus discursos son performativos, realizativos (Austin, 
2008). Entendemos aquí el concepto de “institución” en el sentido sociológico clásico: un orden social estabilizado en el nivel de expectativas reflexivas de acción, en donde un actor espera de otro que éste tenga expectativas específicas sobre el comportamiento suyo (Parsons, 1988). A la luz de este concepto de institución, es una falacia afirmar, junto con los peritos judiciales que llevaron a cabo el proceso y junto a Gonzalo Rojas F. y otros especialistas en esta asociación, que en el proceso comparecieron brujos, sólo porque la sentencia con que concluye el proceso afirma que al menos dos de los individuos acusados son culpables de delito. Lo que tenemos más bien es un conjunto de documentos que dan cuenta de discursos en los cuales se performa (Callon, 2007) y se hace real la institución de la brujería -entendida como expectativas reflexivas de acción- al afirmar un mundo en el cual ella es posible (Valenzuela Avaca, 2011).

Como se ha señalado, este es un mundo en el cual el cosmos se articula siguiendo las leyes de la reciprocidad, las cuales afectan los fenómenos cotidianos de la superficie como si fuesen fenómenos naturales, incluyendo las acciones y los sufrimientos de las personas. En este mundo, los quiebres de las expectativas normativas de acción requieren una sanción que contrapese el daño realizado. Esta misma sanción es una expectativa generalizada de acción, cuya violación trae consigo vergüenza y difamación, pues con ello se arriesga que las expectativas normativas caigan en el olvido y pierdan su capacidad estructurante o de estabilización del orden social (Cevolini, 2011). De modo que en este mundo puede esperarse del otro -y el otro puede esperar de uno esta expectativa específica- que se actuará en el sentido de la sanción cuando se defrauden las expectativas normativas.

Los documentos judiciales dan cuenta de un mundo en el cual tales expectativas se activan cuando no se tiene certeza sobre la causa natural de dolencias corporales. Es necesario distinguir aquí entre expectativas normativas y cognitivas. Siguiendo a Niklas Luhmann (1996, 2007), se entienden por expectativas normativas aquellas que son mantenidas aún en caso de desilusión, pues se las tiene por "justificadas". Expectativas cognitivas son, en cambio, aquellas que son corregidas en caso de desilusión, como en el caso del conocimiento (Luhmann, 1996: 103). En estos documentos judiciales se observa un mundo en el cual aquellas expectativas de venganza se activan cuando el conocimiento deja de sostener la dolencia en términos de enfermedad. En tal caso se lleva a cabo un procedimiento para aclarar su causa moral: en este mundo, las dolencias pueden ser síntomas o señales de una venganza y, por lo tanto, un índice de que expectativas normativas 
que otro actor tenía sobre el comportamiento propio fueron decepcionadas. No es necesario comprobar que aquel otro actor efectivamente haya sido decepcionado en sus expectativas normativas: ya se ha visto que uno debe esperar de él que lleve a cabo acciones orientadas a sanar el daño causado al orden moral. Intentar comprobarlo sería considerado un oprobio, toda vez que se conoce una razón para que haya actuado como se espera de él. En este mundo, cuando la dolencia corporal no adquiere sentido como enfermedad, se tiene a la mano un procedimiento que permite identificar la violación de las leyes cósmicas de la reciprocidad que le otorgan sentido a la dolencia como rastro de una venganza. La evidencia no hace la conclusión, es la conclusión la que construye su propia evidencia: la enfermedad de todos en el cuerpo propio.

Desde luego, este mundo no es único de estas comunidades que habitaban Chiloéafinesdelsiglodiecinueve, sinoqueparecesercompartidoporsociedadesorales, donde "...cualquier evento positivo puede ser interpretado como una recompensa, cualquier evento negativo como un castigo por una culpa que uno debe expiar" $2^{2}$ (Cevolini, 2011: 73). Se puede ver que esta institución, cuyos rastros encontramos en los documentos judiciales, tiene un carácter eminentemente moral: de reforzamiento de las leyes morales que, como un equivalente funcional de la causalidad, dan sentido y orden al cosmos (Cevolini, 2011). Ello queda claro en la semántica utilizada en el proceso legal, que describe las acciones de los individuos como buenas o malas: como acciones que son intrascendentes para la institución de los brujos o como "maldades" que es preciso corregir a través de la magia, con ayuda de técnicas de adivinación.

Atribuir culpabilidad a quien es señalado, como resultado de la aplicación de técnicas adivinatorias, como el autor de la venganza -quien actúa en nombre del afectado-, equivale a reafirmar y hacer real este mundo. Esto es justamente el rol que jugaron los autores del proceso judicial. Del otro lado de la institución de la brujería nos encontramos con una segunda institución que se especializa -o prioriza como problema de referencia (Luhmann, 2007)- la estabilización de expectativas normativas de comportamiento. A diferencia de la hechicería que se atribuye a los brujos, tenemos de este lado una red de decisiones legales recursivas que interpretan los eventos singulares a la luz de decisiones legales anteriores. Es decir, una institución cuyas operaciones señalan las acciones cotidianas ya sea como confirmación o como decepción de expectativas normativas que han sido

2 Las traducciones son mías, salvo que se indique lo contrario en el listado bibliográfico N.A. 
codificadas en un cuerpo legal que tiende a la coherencia interna - integración por consistencia de pautas, en el sentido de Talcott Parsons (1988).

En lugar del principio de equivalencia que guía las operaciones del "Tribunal de la Raza” en un contexto de oralidad primaria, esta segunda institución se orienta por el principio de igualdad: en lugar de generar una ilusión de equivalencia entre las partes de la sociedad -los segmentos y sistemas de parentesco- por medio de una estructura de don y devolución que se pierde en el tiempo, la aplicación de la ley se vuelve sobre sí misma asegurando coherencia en sus operaciones, de modo que iguales casos son tratados de igual manera (Cevolini, 2011). A diferencia de la hechicería que se atribuye a brujos, podemos comprender esta institución a muy grandes rasgos basándonos en la observación luhmanniana del derecho como un subsistema funcional de la sociedad (Luhmann, 2002, 2007). Este es un sistema comunicacional que se diferencia en el medio de la validez a través de la distinción entre comportamiento (referencia externa de la comunicación) y regla (auto-referencia de la comunicación). La unidad de esta distinción se torna operativa para el mismo sistema (podría decirse que se desparadojiza) por medio de la diferenciación de un código binario que observa la distinción entre comportamiento y regla en términos de legalidad e ilegalidad. Así, este sistema reconstruye el mundo en términos de legalidad: todo comportamiento tiene que poder ser indicado en uno de los lados del código, ya sea como legal o como ilegal. Con ello la justicia se ha vuelto positiva: la legalidad de la acción se define de manera recursiva por mera referencia a códigos legales, perdiendo los puntos de apoyos "naturales" y "cosmológicos". Por ello la acción ilegal no es necesariamente una acción mala. Así, ya no podría decirse, junto con los "reparadores" de Chiloé, que las personas deben ser reparadas -en el sentido de "vigiladas"- para que no hagan "maldades". Tanto la moralidad como las normas de reciprocidad en que se fundamenta aquella institución de la brujería en Chiloé quedan recluidas al entorno del sistema legal, y sólo son observadas desde las definiciones del sistema del derecho.

Mi hipótesis no es, sin embargo, que es posible observar en este proceso legal de 1880 las operaciones autopoiéticas de un sistema funcional del derecho . Pretendo mucho menos. Me centraré en el lado del Tribunal de la Raza Indígena, y sólo podré ver de reojo la interacción entre estos dos modos de enfrentar el problema de la contingencia e inestabilidad de las expectativas normativas de acción según se presentan en la selección de actas que realizó Ramón Espech (1960). Del total 
de declaraciones, Ramón Espech seleccionó aquellas que corresponden a brujos que fueron hallados culpables de algún delito ordinario. Como es de esperar, los documentos son ricos en descripciones de la brujería: de los comportamientos que quieren ser vistos, a la luz de la regla, como legales o ilegales. Encontramos menos descripciones sobre la configuración de la institución del derecho, lo que es una buena razón para dejar este segundo lado en la sombra, aunque no ignorado.

\section{EL TRIBUNAL DE LA RAZA INDÍGENA}

El siguiente esquema pretende resumir la estructura de distinciones que sostiene el funcionamiento del "Tribunal de la Raza Indígena”.

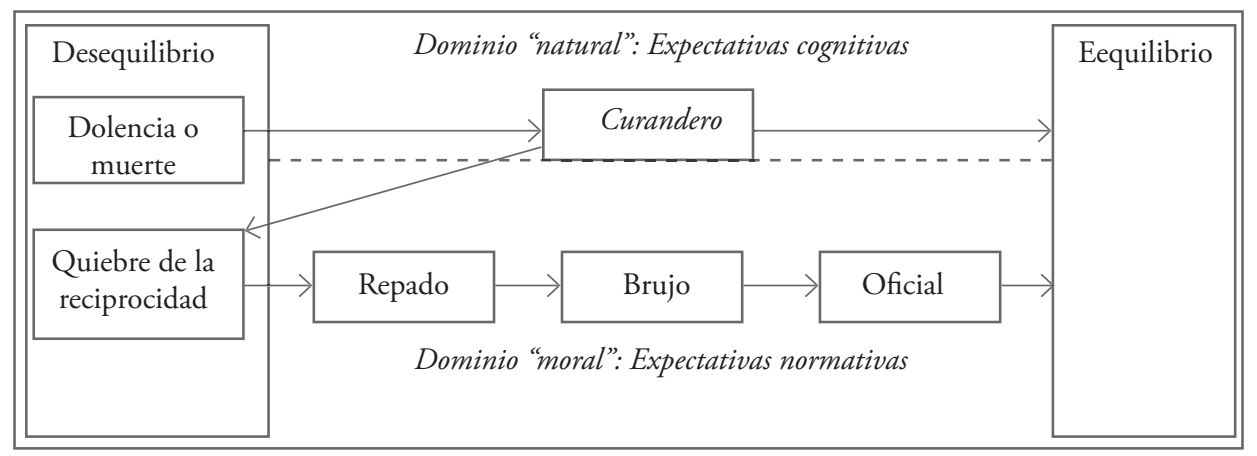

(Esquema de elaboración propia)

He propuesto que la función de esta institución puede comprenderse en términos de una restauración del equilibrio de un cosmos que se articula siguiendo las leyes de la reciprocidad. Esta restauración puede lograrse en dos dominios distintos, dependiendo de las cadenas causales involucradas - distinción marcada en el esquema por la línea discontinua: existe un primer dominio, que he llamado "natural", donde se busca sanar la dolencia en el supuesto de que no es una señal de venganza social, y está el dominio opuesto, que he llamado "moral", en donde tal supuesto se activa. En ambos casos se busca pasar de un estado de desequilibrio del cosmos a uno de equilibrio.

En numerosas ocasiones los documentos del proceso a los brujos de Chiloé se detienen en el fenómeno que fuera investigado por el Tribunal de la Raza: "Merimañ y Quinchipane trataban de quitar la vida a Eusebio de Pelo en Tucoigüe porque tenían muchos víveres y no les daban a ellos” (Espech, 1960: 129); “... porque aquélla le había quitado su marido” (Espech, 1960: 130); “...de que éste 
tenía muchos víveres y les cobraba mucho cuando le cobraban" (Espech, 1960: 130); “Carimonei tenía disputas con la familia de su mujer por terrenos" (Espech, 1960: 131); “...para vengarse de la idea que tenía que dicho Alvarez había tenido malas relaciones con su mujer” (Espech, 1960: 140). La enfermedad y la muerte son males que pueden ser atribuidos a alguien que ha sido afectado por una decepción de expectativas normativas de acción, y por lo tanto se cree que actúa en venganza. Lo mismo encontramos en un listado de demandas interpuestas ante un brujo:

\footnotetext{
"Presenció las demandas que ante Güichapane interpusieron Ignacio Díaz porque decía que Pedro Chiguai le había robado una sabanilla y atribuía al mismo un pasquín que había recibido la noche antes en que lo amenazaban de muerte; Melchor Soto para que le den un médico que reconozca a su mujer que estaba enferma; Fernando Santana para que sepa el ladrón que le había robado un chancho y unas labijas [sic] de molino; Santiago Mayorga para que sepa el ladrón que le comió como veinte ovejas; Pedro Cárcamo para que alcance al brujo que le había aplicado un mal crónico que tenía en la nariz y en la garganta; Agustín Mella para que le alcance a descubrir el brujo que le había envenenado o enfermado a su mujer, dejándola tullida; Rosa Vera para alcanzar a descubrir el brujo que le había enfermado una hija que tenía; Juan Ignacio Avendańo para el mismo objeto por la enfermedad de su mujer y José Patricio Curriman con el mismo fin por enfermedad de su hermano" (Espech, 1960: 142).
}

Aquí se observa con claridad que la enfermedad es vivida como un "mal tirado", obra de un brujo que es "autor" de las muertes: "Calculando que su mujer hubiera sido envenenada, vio a Domingo Cońuecar, que era Presidente de la Recta Provincia, para que le confesara quién era el que había hecho la muerte" (Espech, 1960: 143). La muerte ha sido hecha, y el autor puede ser reconocido por medio del "arte".

Antes de internarnos en este "arte" de los brujos, debemos detenernos en los roles o papeles que componen esta asociación. En la cita anterior se hace referencia al médico, al reparador y al brujo. Este último es un manipulador de símbolos numinosos, por medio de los cuales se interna del lado desconocido, profundo e invisible de la realidad. Este acceso a lo numinoso lo vuelve tabú, como observamos en una declaración del proceso a los brujos de Chiloé: “...los brujos es una gente que está fuera del poder de los demás hombres (de la gente limpia, dice)" (Espech, 1960: 146). La distinción se traza entre gente limpia y sucia: en concordancia con lo señalado por Cazeneuve (1971), los brujos son gente sucia pues entra en contacto con lo numinoso. Los médicos son también nombrados 
como machis o curanderos. Con ello se sitúan fuera de las cadenas de causalidad que pueden ser manipuladas por el lego.

Bajo la instrucción del brujo se haya el reparador. Entre sus atribuciones se encuentra el nombramiento de los médicos y la vigilancia de la población, especialmente de los inmigrantes. Estos últimos son aquellos respecto a quienes no pueden estabilizarse las expectativas de comportamiento en términos de estima: son los extraños (Stichweh, 2010). Estas atribuciones las encontramos en una declaración: "Que hace año y medio, Antonio Nauto, en unión de Mateo Cońuecar, lo nombraron de 'Reparador sobre la tierra' con el objeto de que repare a las personas para que no cometan maldades, para nombrar médicos en los pueblos, y para vigilar a los que llegaran de otras partes a su pueblo" (Espech, 1960: 136). Se entiende que los reparadores hacen justicia, siendo la asociación de brujos un tribunal: "A todos los expresados les dio su nombramiento y son una especie de inspectores que entienden también de justicia" (Espech, 1960: 136). Es interesante notar que la misma denominación de "reparador sobre la tierra" se sostiene en la distinción entre lo asequible y lo inasequible. Este reparador se mantiene del lado de lo asequible. Es el brujo quien une ambos mundos. En este mundo, sobre la tierra, las personas deben ser reparadas para que no cometan maldades.

Los oficiales, en tanto, son obligados por los brujos a llevar a cabo sus resoluciones. Ellos pueden, fuera de tal rol, ser médicos, machis o curanderos, como encontramos en la declaración de un oficial:

"No había cumplido más orden que la que lleva dicha y él de su cuenta no ha envenenado a nadie, pues aún cuando también es curandero, aplica sus remedios determinados que no pueden llegar a causar la muerte; así, por ejemplo, la usa para uso externo contra enfermedades del aire, la piedra agua para la pulmonía, la piedra de ara para colocarla en todos los remedios como cosa que tenía una virtud especial y la cual la usaba poniendo de ella una dosis de polvo, y por último, la pepita de San Ignacio como calmante" (Espech, 1960: 133).

Este oficial sólo ha aplicado veneno cuando un brujo lo ha obligado a hacerlo, y cuando el brujo mismo ha preparado tal remedio. Por su cuenta, es decir, por voluntad propia y en base a su propia sabiduría, el declarante es curandero y no brujo: sana las enfermedades que no son causadas por la voluntad de otro, manteniéndose en el primer dominio antes reconocido. Así, por ejemplo, puede sanar las enfermedades del aire. Por ello, a diferencia de lo propuesto por Rojas F. 
(2002), me parece que debe hacerse una distinción entre el curandero y el brujo, aun cuando ambos roles puedan coincidir también en una misma persona. Sólo en caso de que la enfermedad no cure, se trata de una cárcel, y por lo tanto debe ser derivada a un brujo por medio del reparador para que un oficial lleve a cabo la sentencia. Esta enfermedad consiste en la restricción de la voluntad de la persona enferma por parte de una voluntad ajena: "Dio una orden por escrito a Sánchez para que Parancán notificara a esa mujer, suspenda de la cárcel a dicha enferma..." (Espech, 1960: 144). O en otra carta que se dirigía a un reparador, y fue llevada como prueba en el proceso de 1880: “...y quiero saber si por cual persona está padeciendo mi hijo, mi persona y la persona de mi seńora y todos mis haberes de mi casa y así es que pido bien me lo haga convalecer o pague con cárcel así como padece mi hijo" (Espech, 1960: 148).

Vemos por lo tanto que la enfermedad y la muerte pueden ser atribuidas a una voluntad, generalmente en la forma de la venganza. En ello vemos la característica principal de esta institución: el que se especialice en la desilusión de expectativas normativas de acción. De los brujos se espera que lleven a cabo la venganza, o más bien la ordenen, buscando reparar tal desilusión. Y por lo mismo se espera de los brujos que atribuyan enfermedades o muertes a otros brujos que actúen en nombre de personas "limpias" que buscan venganza, reforzando la creencia en sus capacidades: "Calculando que su mujer hubiera sido envenenada, vio a Domingo Cońuecar, que era Presidente de la Recta Provincia, para que le confesara quién era el que había hecho la muerte” (Espech, 1960: 143). Es interesante también que la relación con los brujos es siempre mediada, en ambos sentidos: hacia quien consulta (por medio del reparador) y hacia quien sufre su acción (por medio del oficial).

En este proceso se necesita que la atribución realizada por el brujo sea entendida como una interpretación del orden de las cosas, y no como una voluntad. Con ello las palabras del brujo adquieren potencialidad performativa o realizativa (Austin, 2008; Callon, 2007; Ramos Zincke, 2012): crean una realidad que se opone a las voluntades. De lo contrario, la misma brujería se volvería sujeto de represalias, lo que finalmente ocurrió en el caso judicial de 1880, en un mundo que ya no se corresponde con aquella institución. Según observa Cevolini (2011: 69), este problema, que es característico de cualquier proceso de conflicto gatillado por la violación de expectativas normativas, puede ser confrontado por técnicas adivinatorias altamente formales. Esto es también lo que encontramos en 
las actas del proceso contra los brujos de Chiloé: la referencia a procedimientos de manipulación de símbolos, cuyos resultados son "leídos" por el brujo. En ello se hermanan las técnicas adivinatorias y las modernas técnicas de la ciencia (incluyendo desde luego aquellas que sirven al poder judicial): en ambos casos las enunciaciones son modalizadas para adquirir potencialidad performativa por medio de la división del mundo entre la enunciación y el objeto enunciado al cual ella se acomoda (Callon, 2007; Latour y Woolgar, 1986).

Pero a diferencia de las técnicas de la ciencia, las de adivinación y magia requieren del secreto (Lévi-Strauss, 1987). A diferencia de las sociedades donde se legitiman los procedimientos siguiendo una racionalidad formal (Kalberg, 1980; Weber, 1984), las sociedades de cultura oral pueden recurrir al secreto como mecanismo que reduce las posibilidades que son abiertas por el código de aceptación y rechazo presente en toda comunicación: “...los problemas de la comunicación se resuelven, o cuando menos se estructuran, a través de la represión de la comunicación" (Luhmann y De Giorgi, 1998: 100). El conocimiento de las cosas sacras queda reducido a un grupo reducido de individuos, iniciados en ritos igualmente sacros. De esta manera, el secreto protege a estas comunicaciones del rechazo. Por otro lado, la institución de tal secreto tiene por resultado, “...una desconfianza organizada a lo largo de esta línea principal que separa a los que saben de los que no, y que diferencia a la sociedad" (Luhmann y De Giorgi, 1998: 100). La sociedad se divide entre los que saben y los que no saben; entre los brujos y la gente limpia. Quizá aluda a este hecho el título de "La Mayoría" que recibe la asociación de brujos. Como señala Jean Cazeneuve (1971), quienes han sido iniciados en el conocimiento secreto alcanzan plenamente la mayoría de edad, se convierten en hombres.

Como fuere, en las actas del proceso contra los brujos de Chiloé encontramos alusiones a una sabiduría constituida por técnicas secretas de lectura de los movimientos de las profundidades de la realidad. Esto es especialmente notorio cuando se hace referencia al "chayanco" y al "Macuñ":

\footnotetext{
"Llaman a lo primero una piedra cristalina que también designan con el nombre de mapa y que sirve para distinguir a los que son brujos. Ellos le dan este poder y el declarante ignora de donde las han adquirido sus compañeros que lo tienen. Lo segundo es una parte de la piel de los brujos que mueren y los que quieren tenerla la sacan en el panteón a la izquierda del cuerpo en dirección del pecho hacia la barriga. Esa piel la curten con ciertas yerbas y en seguida los brujos se la cuelgan con unos cordones al lado izquierdo y con ella andan de noche produciendo una luz especial que los distingue (dice aquí que no sabe explicar la razón de este fenómeno)" (Espech, 1960: 129).
} 
Según se desprende de otras declaraciones, el "chayanco" o "mapa del arte" no sirve para distinguir a los brujos en el sentido de un símbolo de pertenencia, sino más bien como técnica adivinatoria para reconocer al brujo que ha sido autor de las muertes o las enfermedades que se investigan, y la persona que ha solicitado al brujo su intervención:

"Así estaban cuando Guaquin hizo reunir a toda la familia de Colipichún y en seguida les hizo jurar que no divulgaran lo que iban a ver, amenazándoles con una multa excesiva si faltaban. Tomó un vaso con agua y dentro de él puso una piedra que no se fijó de qué clase sería, siendo Panichine el que hizo esto y éste comenzó a romancear en veliche, fijándose en el vaso así como que trataba de descubrir a la persona que tenía enfermo a Colipichún"(Espech, 1960: 148-149).

Tal piedra es el "chayanco" o "mapa del arte". Me interesa recalcar dos aspectos de esta técnica. Primero, que ella permite "reconocer" al autor de la enfermedad, de manera que el enunciado del brujo se comprende en el sentido de una adecuación a la realidad, en lugar de una creación de una realidad. Con ello se refuerza su carácter performativo. En segundo lugar, en esta cita se observa claramente que esta institución se caracteriza por la instauración del secreto. En este mismo sentido, en otra declaración en la que se narra el rito de pasaje por medio del cual una persona se convierte en reponedor (¿reparador?), encontramos: “...en seguida le hicieron hacer la promesa de no decir nada de lo que viera, de no divulgar los secretos...” (Espech, 1960: 127).

El principal secreto es "el arte", simbolizado por el libro que recibió la hechicera Chillpilla de manos de Moraleda, según los relatos orales acerca de la fundación de la asociación de brujos:

"Que por tradición y por habérselo oído a su padre y a otros más que ya son muertos sabe que en un tiempo de que no se tiene noticia, pero ya en la dominación española, llegó a Payos en un buque de esa nación un individuo apellidado Moraleda con el objeto de conseguir algunos naturales para llevar a la península. No consiguió ninguno en ese lugar, por cuya causa se vino a Tenaun, donde tampoco encontró indios que lo siguieran. Ahí en ese punto se presentó Moraleda haciendo ver que era hechicero transformándose en pescado, lobo, palomas y otros animales y mostrando con ello que por tal causa debían seguirlo los indios. Casualmente en el mismo punto había una mujer llamada Chillpila residente en Quetalco, que tenía fama de hechicera y los mismos indios buscáronla para hacerla competir con Moraleda. Entre las varias pruebas que hizo ésta, consiguió dejar en seco el buque de Moraleda en el mismo punto donde se hallaba anclado, y después ponerlo a flote. Moraleda con esto se dio por vencido y en señal de reconocimiento, regaló a Chillpila un libro de hechicerías para que enseñara a los demás indígenas. Moraleda 
se retiró de ahí, recalando a Quicaví como de paso, y dejando a este mismo lugar con el nombre de España y Lima. La Chillpila llevó el libro a Quicaví para que aprendieran los indígenas y de ahí se organizaron las asociaciones en que ahora figura el declarante" (Espech, 1960: 127).

En relación con la institucionalización del secreto, es importante notar que tal libro que deja Moraleda parece ser más un símbolo del "arte" que un portador de "información". Aquel "arte" debe mantenerse en secreto y su símbolo es celosamente guardado bajo llave en una cueva cuya ubicación sólo conocen los miembros de la "Recta Provincia". En diversas declaraciones se completa la narración señalando que aquella llave, por ser de latón, se derritió en un incendio, perdiéndose definitivamente el acceso a la cueva o "despacho" de Quicaví con su Trono, su Libro de Justicia, y sus guardianes.

Aquello que es secreto es sobre todo una técnica adivinatoria que otorga capacidad performativa a los enunciados. Supuesto en ello es que los brujos cuentan con instrumentos que facilitan a los oficiales para que lleven a cabo sus decisiones: los llamados "remedios", como el que se describe en el siguiente fragmento:

\footnotetext{
"Bocado es un remedio que usan los brujos para causar a los hombres una enfermedad que les hincha la barriga, que les da mucha sed, muchos vómitos, sin que les permita parar nada en el estómago y que los aniquila por completo hasta que llegan a morir. Ese remedio lo hacen de lagartijas y sapos que secan al sol y después en una pequeńa narigada le ponen el polvo en alguna bebida que se quiere dar a la víctima. Suelen llegar a restablecerse de esta enfermedad, aplicando las mismas lagartijas y sapos tostados con sal también en una pequeńa narigada y tomando esto con agua bendita. Así en una semana puede restablecerse" (Espech, 1960: 135).
}

Como ésta, una lista de doce remedios se encuentra en la declaración citada de Aurora Quinchén Agüil (Espech, 1960: 134-136). La diferencia entre el remedio y su antídoto está marcada por la presencia de agua bendita, introduciendo un corrector que escapa nuevamente del ámbito de las voluntades humanas, reforzando nuevamente la performatividad de los enunciados.

\section{CONCLUSIÓN}

En los documentos del proceso contra los brujos de Chiloé se observa que se espera que los brujos ordenen la realización de acciones de venganza frente a expectativas normativas (expectativas que no son corregidas en caso de desilusión), $\mathrm{y}$ en especial frente a violaciones de las normas de reciprocidad. Tales acciones se 
traducen en enfermedades o muertes. Por lo mismo, se espera de ellos que puedan "reconocer" los autores de enfermedades o muertes cuando ellas no son atribuidas a una voluntad humana, pues alguien le ha querido mal y, consiguientemente, le ha hecho o tirado un mal.

Ante dudas acerca del dominio de causalidades que puede otorgar sentido a la enfermedad o a la muerte, se puede recurrir a un "curandero", un "médico" o un "machi", quien tiene el saber de las causas de la naturaleza no humana. Si el enfermo no sana, y por lo tanto la expectativa debe ser corregida (y en ese sentido hablamos de expectativas cognitivas, a diferencia de las expectativas normativas), la enfermedad puede ser atribuida a la voluntad de algún individuo que se asume movido por la envidia o por deseos de venganza. En una sociedad en que la reciprocidad fundamenta las principales expectativas normativas, la envidia puede ser causa de venganza, ya que no se ha recibido lo que ha sido debido.

Cuando la enfermedad o la muerte son atribuidas a voluntades, se recurre a un "reparador", quien recibe la demanda correspondiente y se la entrega al "brujo" de la región. Éste es una persona que ha sido iniciada en artes secretas. Sobre todo, tiene poder sobre las personas: puede causar enfermedad y muerte, y controlar la voluntad. Como se ha señalado, se espera del brujo que pueda reconocer, por medio de su arte, quién es el autor de la enfermedad o de la muerte que se investiga y qué corresponde hacer. El enunciado que el brujo pronuncie (o que se le atribuya a él) debe ser modalizado de manera tal que se comunique su adecuación a una realidad objetiva que está fuera de su voluntad. A partir de esto, el brujo ordena al "oficial” llevar a cabo su resolución con los medios que él le entrega: por lo general se envenena al autor, causando su enfermedad o su muerte, lo que podría reiniciar el ciclo en caso de que su enunciado termine por no gozar de un nivel suficiente de facticidad.

Si desde aquí volvemos a Ramón Espech, del otro lado de la distinción, se abre un abismo. Él ha escrito: “...conversando sobre los brujos, supe que desde la persecución que les hizo el intendente, curan sólo a escondidas; que sus yerbas no tienen poder contra él; y que hacen mucha falta para curar los males tirados" (Espech, 1960: 126). Tres cosas sabe Ramón Espech: primero, que la persecución de la cual han sido objeto los brujos ha dejado a éstos en la ilegalidad: curan sólo a escondidas. Segundo, que el arte de los brujos no tiene poder sobre el intendente. Y tercero, que los brujos siguen haciendo mucha falta para curar los males tirados. Esto último nos indica que la persecución legal de la brujería no 
habría logrado erradicar el universo de sentido en el cual aquella institución se sostenía. Sin embargo, a los brujos se les presenta una realidad social en la cual sus medios mágicos no tienen eficacia alguna.

\section{REFERENCIAS}

Álvarez Sotomayor, Agustín. "Los brujos de Chiloé", Archivos de Folklore Chileno 67 (1954): 89-106.

Anónimo. Los brujos de Chiloé: Célebre proceso del Juzgado de Ancud: declaraciones de los reos. Santiago de Chile: Casa Editorial de Ponce Hermanos, 1908.

Austin, John Langshaw. Cómo hacer cosas con palabras y acciones. En G. R. Carrió y E. A. Rabossi, trads., J. O. Urmson, (Ed.), Buenos Aires: Paidós, 2008.

Balbontín Moreno, Manuel. Brujos y hechicerías. Santiago de Chile: Ediciones Arcos, 1965.

Barrientos, Javier. "Nuevos antecedentes del proceso a los brujos de Chiloé. 18801881”, Chiloé, Revista de Divulgación del Centro Chilote 9 (1988): 3-8.

Callon, Michel. "What does it mean to say that economics is performative?". En Donald MacKenzie, Fabian Muniesa, y Lucia Siu (Eds.), Do economists make markets? On the performativity of economics. Princeton: Princeton University Press, 2007.

Cárdenas, Renato; Montiel Vera, Dante y Hall, Catherine. Los Chono y los Veliche de Chiloé. Santiago de Chile: Ediciones Olimpho, 1991.

Cárdenas, Umiliana. Casos de Brujos de Chiloé. Santiago de Chile: Editorial Universitaria, 1989.

Cavada, Francisco Javier. Chiloé y los chilotes: Estudios de folklore y lingüistica de la provincia de Chiloé (República de Chile), acompañados de un vocabulario de chilotismos y precedidos de una breve Reseña Histórica del Archipiélago. Santiago de Chile: Imprenta Universitaria, 1914. 
Cazeneuve, Jean. Sociología del rito. Buenos Aires: Amorrortu, 1971.

Cevolini, Alberto. "Erynnerung. Evolution and semantics of primitive law". En Ignacio Farías y José Ossandón (Eds.), Comunicaciones, semánticas y redes. Usos y desviaciones de la sociología de Niklas Luhmann. México: Universidad Iberoamericana, (2011): 63-83.

Contreras, Constantino. "Mitos de brujería en Chiloé. (Estudio LingüísticoFolklórico)”, Estudios Filológicos 2 (1966): 161-198.

Espech, Ramón. "Proceso a los brujos de Chiloé", Anales chilenos de historia de la medicina I (1960): 124-162.

Flores, Máximo. “Sociedad y brujería en Chiloé, 1850-1900”. Tesis de Licenciatura en Historia. Chile: Pontificia Universidad Católica de Chile, 1994.

Kalberg, Stephen. "Max Weber's Types of Rationality: Cornerstones for the Analysis of Rationalization Processes in History", The American Journal of Sociology 85/5 (1980): 1145-1179.

Latour, Bruno; Woolgar, Steve y Salk, Jonas. Laboratory Life: The Construction of Scientific Facts. Durham: Duke University Press, 1986.

León, Marco Antonio. La cultura de la muerte en Chiloé. Santiago de Chile: RIL Editores, 2007.

Lévi-Strauss, Claude. Antropología estructural. Barcelona: Paidós, 1987.

Luhmann, Niklas. Social Systems. (J. Bednarz y D. Baecker, trads.), Stanford University Press, Stanford (California), 1995.

La ciencia de la sociedad. (S. Pappe, B. Erker, y L. F. Segura, trads.), México: Universidad Iberoamericana - Anthropos, ITESO, 1996.

. Die Gesellschaft der Gesellschaft. Suhrkamp, Frankfurt am Main, 1997.

El derecho de la sociedad. México: Universidad Iberoamericana, 2002. 
--o-c-o----. La sociedad de la sociedad. México: Herder - Universidad Iberoamericana, 2007.

Luhmann, Niklas y De Giorgi, Raffaele. Teoría de la Sociedad. (M. Romero Pérez y C. Villalobos, trads.) (Ed. Rev.). Mexico: Universidad Iberoamericana Triana Editores, 1998.

Mancilla Pérez, Luis. Chiloé: brujos y machis en la Recta Provincia (origenorganización-rituales-maleficios y medicina). Ancud, 1994 a.

-----------. La magia del pais de los chilotes. Castro: Ed. "La Ciudad de los Césares», C, $1994 \mathrm{~b}$.

Marino, Mauricio y Osorio, Cipriano. Chiloé, cultura de la madera: proceso a los brujos de Chiloé. Ancud: Imprenta Cóndor, 1987.

Mascareño, Aldo. Diferenciación y contingencia en América Latina. Santiago de Chile: Ediciones Universidad Alberto Hurtado, 2010.

Mauss, Marcel. A General Theory of Magic. London: Routledge, 2001.

. Essai sur le don: Forme et raison de l'échange dans les sociétés archaïques. Paris: Presses universitaires de France, 2007.

Parsons, Talcott. The System of Modern Societies. Englewood Cliffs, NJ: PrenticeHall, 1971.

Parsons, Talcott. La sociedad. Perspectivas evolutivas y comparativas. México: Editorial Trillas, 1974. El sistema social. Madrid: Editorial Alianza, 1988.

Ramos, Claudio. El ensamblaje de ciencia social y sociedad. Santiago de Chile: Universidad Alberto Hurtado, 2012.

Rojas Flores, Gonzalo. Reyes sobre la Tierra: brujería y chamanismo en una cultura insular: Chiloé entre los siglos XVIII y XX. Santiago de Chile: Editorial Biblioteca Americana, 2002. 
Romo Sánchez, Manuel. (Comp.), Aves y plantas en la brujería de Chiloé. Santiago de Chile, 1987.

(Ed.), Diccionario de la brujería en Chiloé. Santiago de Chile, 1989.

Diccionario de brujería de Chiloé. Santiago de Chile: Platero Ediciones, 1997.

Serres, Michel. The Parasite. (L. Schehr, trad.), Minneapolis - London: University of Minnesota Press, 2007.

Stichweh, Rudolf. Der Fremde. Studienzu Soziologie und Sozialgeschichte. Suhrkamp Verlag, Frankfurt am Main, 2010.

Valenzuela Avaca, Eduardo. "Rei de los brujos: introducción y transcripción del documento: Proceso a Domingo Rojas, mulato, por hechicero y vago. 1765”, Universum 26/1 (2011): 139-150.

Weber, Max. Ensayos sobre sociología de la religión. (J. Almaraz y J. Carabaña, eds.). Taurus, Buenos Aires, 1984, (Vols. 1-3, Vol. 1). 